\title{
Editorial
}

\section{Editorial to Cancer Biomarkers vol. 32}

Cancer Biomarkers was launched in 2005 to share new research and discoveries on the use of biomarkers in the detection, diagnosis, and treatment of cancer in its various forms. Since then, the journal has grown in size, quality, and credibility. During its more than 15 years, Cancer Biomarkers has published 31 volumes filled with articles by authors from all corners of the planet. The journal is globally distributed and has its articles frequently cited by researchers in peer-reviewed journals each year. The great recognition of the importance of Cancer Biomarkers to the scientific community reflects on its metrics, including the Journal Impact Factor, which reached 4.388 in 2020.

We want to express our gratitude to the community including contributors of high quality research work and reviewers who provide valuable, objective assessment of submitted manuscripts. Our stellar Editorial Board is the face of the journal's incredible accomplishments. The reputable scientists composing Cancer Biomarkers Editorial Board are the basis of our peer review process, along with ad hoc reviewers who contribute their knowledge to enhance and ensure the high quality of papers published in the journal. Most of all, we thank all authors who choose to publish their articles in Cancer Biomarkers. It is an honor to be selected among so many journals as the home for the latest research findings. Finally, our sincere appreciation goes to the readers who trust our journal as a source of reliable and accurate scientific information.

The field of cancer biomarkers has expanded in the past decades. From promising tools, biomarkers have developed into powerful allies to fight cancer on several fronts, including risk assessment, diagnosis, prognosis, and development of drugs and treatments. Each day we take a step further into the final goal of our research efforts: to reduce cancer mortality and morbidity at the lowest possible level. 